\title{
LAGUERRE GEOMETRIES AND SOME CONNECTIONS TO GENERALIZED QUADRANGLES
}

\author{
MATTHEW R. BROWN \\ (Received 15 November 2005; revised 30 September 2006) \\ Communicated by L. Batten
}

\begin{abstract}
A Laguerre plane is a gennetry of points. lines and circles where three pairwise non-collinear points lie on a unique circle. any line and circle meet uniquely and finally. given a circle $C$ and a point $Q$ not on it for each point $P$ on $C$ there is a unique circle on $Q$ and touching $C$ at $P$. We generalise to a Laguerre geometry where three pairwise non-collinear points lie on a constant number of circles. Examples and conditions on the parameters of a Laguerre geometry are given.

A generalized quadrangle (GQ) is a point. line geometry in which for a non-incident point, line pair $(P, m)$ there exists a unique point on $m$ collinear with $P$. In certain cases we construct a Laguerre geometry from a GQ and conversely. Using Laguerre geometries we show that a GQ of order $\left(s, s^{2}\right)$ satisfying Property $(\mathrm{G})$ at a pair of points is equivalent to a configuration of ovoids in three-dimensional projective space.
\end{abstract}

2000 Mathematics subject classification: primary 51E20. $51 \mathrm{E} 12$.

\section{Laguerre planes}

The study of Laguerre planes is motivated by the study of the quadratic cone in the projective space $\operatorname{PG}(3, \mathbb{F})$ over the field $\mathbb{F}$. The quadratic cone is formed by taking a cone with point vertex over an irreducible quadric (conic) in a plane and with canonical equation $X_{0}^{2}+X_{1} X_{2}=0$. Laguerre planes axiomatise the point, line and plane section geometry of the quadratic cone.

A Laguerre plane $\mathscr{S}=(\mathscr{P}, \mathscr{L}, \mathscr{C})$ is an incidence structure of points, lines and circles, respectively, such that the following axioms are satisfied.

This work was supported by the Australian Research Council

(C) 2007 Australian Mathematical Society 1446-7887/07 \$A2.00+0.00 
(L1) Three pairwise non-collinear points are incident with a unique circle.

(L2) For any circle $C$, point $P \in C$ and point $Q$ not collinear to $P$ there is a unique circle $D$ incident with $Q$ and touching $C$ at $P$ (that is, $C$ and $D$ are incident with exactly one common point $P$ ).

(L3) A point is incident with a unique line and a line meets a circle in a unique point.

(L4) Every circle is incident with at least three points and there exists more than one circle.

For more on Laguerre planes and the more general circle planes see $[3,6]$.

Note that we will often refer to a circle or a line as the set of points with which it is incident.

The touching axiom (L2) has an important consequence. If $P$ is a point on a circle $C$ then any other two circles touching $C$ at $P$ must also touch each other at $P$, or else Axiom (L2) is violated. Hence the circles on a fixed point $P$ are partitioned into sets which touch pairwise at $P$ and partition the points of $\mathscr{S}$ not collinear with $P$. Such a set of circles is called a pencil, and $P$ is called the base point of the pencil.

Naturally, the quadratic cone in PG $(3, \mathbb{F})$ gives an example of a Laguerre plane. In addition, there are other sets of points in projective space that have properties similar enough to those of a quadric in PG $(3, \mathbb{F})$ that they may be used in the construction of Laguerre planes and, as we shall see later, the more general Laguerre geometries.

An ovoid ([29], see [10]) $\mathscr{O}$ of a projective space of dimension two or greater is a non-empty set of points satisfying the following conditions.

(1) No three points are collinear.

(2) The tangent lines (that is, lines meeting $\mathscr{O}$ in a single point) at a point $P \in \mathscr{O}$ form a hyperplane.

Note that in two dimensions an ovoid is usually called an oval.

For an ovoid in three dimensions or greater, any hyperplane of the space that is not tangent to the ovoid must intersect the ovoid in an ovoid of that hyperplane.

We can construct a Laguerre plane from an oval in the following manner. Form a cone in three dimensions from a point vertex over an oval in a plane and then take the non-vertex points, lines and plane sections not containing the vertex as the points, lines and circles of the Laguerre plane.

In the oval cone models for Laguerre planes (which include the quadratic cone) we have a local projective structure in the sense that, for any non-vertex point $P$ on the oval cone, $\operatorname{PG}(3, q) / P$ is a projective plane. Also, if we remove the tangent plane to the oval cone at the point $P$ then in the quotient space we have an affine plane. (Note that in this context a tangent plane meets the oval cone in a single line.) This local or internal structure is preserved by generalising to Laguerre planes. 
If $\mathscr{S}=(\mathscr{P}, \mathscr{C}, \mathscr{L})$ is a Laguerre plane then the derived plane at $P \in \mathscr{P}$ is the point-line geometry $\mathscr{S}_{P}$ with

Points: points of $\mathscr{S}$ not collinear with $P$,

Lines: circles incident with $P$ and lines of $\mathscr{S}$ not incident with $P$, Incidence: inherited from $\mathscr{S}$.

Since three pairwise non-collinear points define a unique circle in $\mathscr{S}$, we have that two points define a unique line in $\mathscr{S}_{P}$ (whether they be collinear or non-collinear in $\mathscr{S}$ ). The touching axiom for $\mathscr{S}$ implies that the circles of $\mathscr{S}$ incident with $P$ fall into parallel classes as lines of $\mathscr{S}_{P}$. The lines of $\mathscr{S}$ not incident with $P$ form a parallel class of lines in $\mathscr{S}_{P}$. Note also that any circle of $\mathscr{S}$ not incident with $P$ meets any circle incident with $P$ in at most two points (Axiom (L1) guarantees this for any two distinct circles). Hence we have the following important and well-known theorem.

THEOREM 1.1. Let $\mathscr{S}=(\mathscr{P}, \mathscr{C}, \mathscr{L})$ be a Laguerre plane and $P \in \mathscr{P}$. Then

(1) $\mathscr{S}_{P}$ is an affine plane and we denote its projective completion $\overline{\mathscr{S}_{P}}$, and

(2) if $C$ is a circle of $\mathscr{S}$ not incident with $P$ then in $\overline{\mathscr{S}_{P}}$ the points of $\mathscr{S}_{P}$ incident with $C$ plus the point of $\overline{\mathscr{S}}_{P}$ that is the parallel class of $\mathscr{S}_{P}$ corresponding to the lines of $\mathscr{S}$ not incident with $P$, form an oval of $\overline{\mathscr{S}_{P}}$.

Note that in all of the models for Laguerre planes mentioned the derived affine plane is classical.

This relationship between Laguerre planes, affine planes and ovals allows many interesting classification/characterisation results for Laguerre planes to be proved and tells us much about the structure of Laguerre planes (for example, see [8]).

\section{Finite Laguerre planes, ovoids and Laguerre geometries}

We now focus our attention on finite Laguerre planes and investigate the links to ovoids in finite projective spaces. This in turn will suggest a natural generalisation of Laguerre planes.

If a finite Laguerre plane $\mathscr{S}$ has a line incident with a finite number $n$ of points, then it is straightforward to show that each line is incident with $n$ points, there are $n+1$ lines, each circle has $n+1$ points, there are $n^{2}+n$ points in total and every derived plane has order $n$. The number $n$ is called the order of $\mathscr{S}$.

THEOREM 2.1 ([29]). Let $\mathscr{O}$ be an ovoid of $\mathrm{PG}(n, q), q$ a prime power.

(1) $|\mathscr{O}|=q^{n-1}+1$.

(2) $n=2$ or 3 . 
Both parts of the above theorem can be proved by straightforward counting arguments. Given the above theorem, we shall often employ the term oval to refer to a two-dimensional ovoid and ovoid to refer to a three-dimensional ovoid.

It is known that if $q$ is odd then the ovoids are always classical, that is an elliptic quadric if $n=3$ and an irreducible conic if $n=2$ ([1, 14,21]). In the case where $q$ is even there are non-classical examples of both ovoids and ovals (see $[9,13])$.

We have seen that a cone over an oval gives rise to a Laguerre plane, so now we will investigate the geometry of a cone $\mathscr{K}$ over an ovoid (with point vertex $V$ outside the three-space of the ovoid). In particular, we will.consider the geometry of points, lines and ovoidal hyperplane sections (that is, where the hyperplane does not contain $V$ ) of $\mathscr{K}$. Firstly, there is a unique line on any non-vertex point. Secondly, any three pairwise non-collinear points define a plane of PG $(4, q)$ which intersects $\mathscr{K}$ in an oval and is contained in $q$ hyperplane sections of $\mathscr{K}$ not containing $V$. This number is finite as we are now working in a finite projective space. Finally, any ovoidal hyperplane section has a unique tangent plane at every point. The hyperplanes about this tangent plane, not containing $V$, give a set of $q$ ovoidal hyperplane sections that meet pairwise in a common point. That is, the tangent plane property of the ovoid means that the geometry of $\mathscr{K}$ satisfies the touching axiom as in Laguerre planes. This geometry prompts the following generalisation of Laguerre planes where we modify only the first axiom.

A Laguerre geometry $\mathscr{S}=(\mathscr{P}, \mathscr{C}, \mathscr{L})$ is an incidence structure of points, lines and circles, respectively, satisfying Axioms (L2)-(L4) of a Laguerre plane and also.

$\left(\mathrm{Ll}^{*}\right)$ Three pairwise non-collinear points are incident with a constant (finite) number $s>0$ of circles.

We will say that $\mathscr{S}$ is finite if $\mathscr{P}$ is a finite set (and hence so are $\mathscr{C}$ and $\mathscr{L}$ ). If a line of a finite Laguerre geometry $\mathscr{S}$ is incident with $n$ points then all lines of $\mathscr{S}$ are incident with $n$ points. The parameter $s$ is the number of circles of $\mathscr{S}$ on three pairwise non-collinear points. We introduce a parameter $\ell$ to denote the number of lines of $\mathscr{S}$, that is $\ell=|\mathscr{L}|$. Given these parameters for $\mathscr{S}$ it is straightforward to count that there are $n \ell$ points in total, $\ell$ points incident with a circle, $n^{3} s$ circles in total, $n s$ circles incident with two given non-collinear points and $n^{2} s$ circles incident with a given point.

As in the Laguerre plane case, Axiom (L2) of a Laguerre geometry means that the circles on a fixed point $P$ are partitioned into sets which touch pairwise at $P$ and partition the points of $\mathscr{S}$ not collinear with $P$. As for Laguerre planes, we will call such a set of circles a pencil and the point $P$ the base point of the pencil.

If a Laguerre geometry $\mathscr{S}$ has parameters $n, s, \ell$, then for the case $s=1$ we have exactly the Laguerre planes, which implies that $\ell=n+1$ and the geometry $\mathscr{S}_{P}$ is an affine plane. If, however, $s>1$, what can we say about the concept analogous 
to that of the derived plane and what can we say about the relationship between the parameters?

If we consider our motivating example of a cone with point vertex and base an ovoid $\mathscr{O}$ of $\mathrm{PG}(3, q)$, then we have a Laguerre geometry $\mathscr{S}$ with parameters $n=s=q$ and $\ell=|\mathscr{O}|=q^{2}+1$. For a point $P$ of $\mathscr{S}$, consider extending the concept of the geometry $\mathscr{S}_{P}$ as used for Laguerre planes. This geometry has points the points of $\mathscr{S}$ not collinear with $P$. Lines are of two types, firstly circles of $\mathscr{S}$ incident with $P$ and secondly lines of $\mathscr{S}$ not incident with $P$. In this case the first type of line is incident with $q^{2}$ points of $\mathscr{S}_{P}$, while the second type of line is incident with $q$ points of $\mathscr{S}_{P}$. This is clearly an unsatisfactory definition, so in the definition that follows we omit the second type of line.

If $\mathscr{S}=(\mathscr{P}, \mathscr{C}, \mathscr{L})$ is a Laguerre geometry and $P \in \mathscr{P}$, define $\mathscr{S}^{P}$, the internal structure of $\mathscr{S}$ at $P$, to be the incidence geometry with

Points: points of $\mathscr{S}$ not collinear with $P$, Hyperplanes: circles of $\mathscr{S}$ incident with $P$, and Incidence: inherited from $\mathscr{S}$.

In the case of a Laguerre plane, $\mathscr{S}^{P}$ is a projective plane with an incident point-line pair removed, by which we mean that all points on this line and lines through this point are also removed. In the case of the Laguerre geometry $\mathscr{S}$ arising from a cone with base an ovoid in PG $(3, q)$, if $P$ is a point of $\mathscr{S}$ then the circles on $P$ come from the hyperplanes of PG $(4, q)$ on $P$ but not on the vertex of the cone, while each point of $\mathscr{S}$ not collinear with $P$ spans a distinct line with $P$. Looking in the quotient space $\operatorname{PG}(4, q) / P$, the geometry $\mathscr{S}^{P}$ is the geometry of $\mathrm{PG}(3, q)$ with a point-plane pair removed (corresponding in $\mathrm{PG}(4, q)$ to the line $P V$ and the hyperplane meeting the cone in $P V$ ). These examples prompt the following definition.

A Laguerre geometry $\mathscr{S}=(\mathscr{P}, \mathscr{C}, \mathscr{L})$ has classical internal structure at $P \in \mathscr{P}$ if $\mathscr{S}^{P}$ is a projective space with a point-hyperplane pair removed.

We now return to the question of the relationship between the parameters $n, s$ and $\ell$ of a Laguerre geometry $\mathscr{S}$. The first question is: do $n$ and $s$ determine $\ell$ ? The following example shows that this is not the case. Let $\mathscr{O}$ be an ovoid of $\operatorname{PG}(3, q)$ and let $\overline{\mathscr{O}} \subset \mathscr{O}$ be such that there is a unique tangent plane to $\overline{\mathscr{O}}$ at each point. In this context, by tangent plane at $P \in \overline{\mathscr{O}}$ we mean a plane incident with $P$ and no other point of $\overline{\mathscr{O}}$. If we form a cone in $\operatorname{PG}(4, q)$ with point vertex and base $\overline{\mathscr{O}}$ then the geometry of (non-vertex) points, lines and hyperplane sections of this cone, not containing the vertex, is a Laguerre geometry with $n=s=q$ and $\ell=|\overline{\mathscr{O}}|$. The unique tangent plane at every point of $\overline{\mathscr{O}}$ ensures that the touching axiom is still valid. So in this construction we see that a given $n, s$ may give rise to Laguerre geometries with various values of $\ell$. More generally, this construction will work if we take the cone over a set of points in $\mathrm{PG}(n, q), n \geq 3$, such that no three are collinear and there 
is a unique tangent plane at each point.

The following theorem gives us a definite relationship between $n, s$ and $\ell$.

THEOREM 2.2. Let $\mathscr{S}$ be a finite Laguerre geometry with parameters $n, s, \ell$. Then $n+1 \leq \ell \leq n s+1$. Equality in the lower bound means $s=1, \ell=n+1$ and $\mathscr{S}$ is a Laguerre plane, which is equivalent to $\mathscr{S}$ having intersection sizes between two circles being $0,1,2$. In the case $\ell=n s+1$ we have $s+1 \mid n^{3}-n, n \geq s$ and the possible intersection sizes for two circles are $0,1, s+1$. Further, in the $\ell=n s+1$ case there exist disjoint circles if and only if $n>s$.

PROOF. To start, we recall that the total number of points is $n \ell$, the total number of circles is $n^{3} s$, the number of circles on a pair of non-collinear points is $n s$ and the number of circles on a given point is $n^{2} s$.

Now let $C$ be a fixed circle of $\mathscr{S}$ and $P$ a fixed point of $C$. There are exactly $n-1$ circles meeting $C$ in exactly $P$ and hence $n^{2} s-n$ circles distinct from $C$ meeting $C$ in $P$ and at least one other point. For any point $Q \in C \backslash\{P\}$ there are $n s-1$ circles distinct from $C$ meeting $C$ in at least $P$ and $Q$. Hence there are at most $(n s-1)(\ell-1)$ circles meeting $C$ in $P$ and at least one other point. Hence $n^{2} s-n \leq(n s-1)(\ell-1)$, which is equivalent to $\ell \geq n+1$. From the count, it follows that we have equality if and only if the possible intersection sizes between two circles are $0,1,2$. This is the case if and only if $s=1$ and, since $\ell=n+1, \mathscr{S}$ is a Laguerre plane.

Let the set of circles distinct from $C$ and meeting $C$ in $P$ and at least one other point be $\left\{C_{1}, C_{2}, \ldots C_{n: s-n}\right\}$ and $t_{i}=\left|\left(C_{i} \cap C\right) \backslash\{P\}\right|$. Then $\sum_{i=1}^{n^{2}-s-n} t_{i}=(\ell-1)(n s-1)$ and the average value of the $t_{i}$ is $\bar{t}=(\ell-1)(n s-1) /\left(n^{2} s-n\right)$. If we count triples $\left(Q, R, C^{\prime}\right)$ such that $P, Q, R$ are distinct pairwise non-collinear points and $C^{\prime} \neq C$ and $P, Q, R \subset C \cap C^{\prime}$, then we have $\sum_{i=1}^{n^{-s-n}} t_{i}\left(t_{i}-1\right)=(\ell-1)(\ell-2)(s-1)$. So now if we calculate $\sum_{i=1}^{n^{2} s-n}\left(t_{i}-\bar{t}\right)^{2}=(\ell-1)(n-1)(n s+1-\ell) / n$ we obtain the inequality $\ell \leq n s+1$.

If we have $\ell=n s+1$ then, by the above, any two circles that intersect in at least two points intersect in exactly $\bar{t}+1=s+1$ points. In this case if, for a fixed circle $C$, we count the triples $\left(P . Q, C^{\prime}\right)$ with $P \neq Q$ and $P, Q \in C \cap C^{\prime}$, then we see that the number of circles. distinct from $C$, meeting $C$ in at least two (and hence exactly $s+1$ points $)$ is $(n s+1) n s(n s-1) /\left(s^{2}+s\right)$. Since this is an integer, we have $s+1 \mid n\left(n^{2} s^{2}-1\right)$ and hence $s+1 \mid n^{3}-n$. Now the total number of circles distinct from $C$ not touching $C$ is $n^{3} s-\ell(n-1)-1=n^{3} s-(n s+1)(n-1)-1$ which is an upper bound for the number of circles meeting $C$ in at least two points. Hence $n^{3} s-(n s+1)(n-1)-1 \geq(n s+1) n(n s-1) /(s+1)$, which simplifies to $(n-s)(n-1) \geq 0$ and hence $n \geq s$. If we have equality then, by the count, there are no circles disjoint to $C$ and the possible intersection sizes between circles is 1 and $s+1$. 
COROLLARY 2.3. Let $\mathscr{S}=(\mathscr{P}, \mathscr{L}, \mathscr{C})$ be a finite Laguerre geometry with parameters $n, s, \ell$. If $\mathscr{S}$ has a classical internal structure at some point $P$ then either

(1) $s=1, \ell=n+1$ and $\mathscr{S}^{P}$ arises from a projective plane with a point-line pair removed; or

(2) $s=n, \ell=n^{2}+1$ and $\mathscr{S}^{P}$ arises from a projective 3-space with a point-plane pair removed.

PROOF. Since $\mathscr{S}^{P}$ is classical, it follows that any two distinct circles of $\mathscr{S}$ on $P$ touch at $P$ or intersect in a constant number of points. In the proof of Theorem 2.2, this means that $t_{i}=\bar{t}$ for $i=1, \ldots, n^{2} s-n$ and so

$$
\sum_{i=1}^{n^{2} s-n}\left(t_{i}-\bar{t}\right)^{2}=\frac{(\ell-1)(n-1)(n s+1-\ell)}{n}=0,
$$

from which it follows that $\ell=n s+1$ and $\bar{t}=s$. Hence the number of points of $\mathscr{S}^{P}$ must be $n \ell-n=n^{2} s$. The projective space giving rise to $\mathscr{S}^{P}$ has order $n$ and so $n^{2} s=n^{k}$ for some integer $k$. By Theorem 2.2, we also have that $n \geq s$, so the only possibilities are $s=1$ and $k=2$ or $s=n$ and $k=3$.

\section{Generalized quadrangles}

Now we introduce a particular class of point-line geometries, the generalized quadrangles, and show their connection to Laguerre geometries. Generalized quadrangles were introduced by Tits in [28].

A generalized quadrangle (GQ) $\mathscr{S}=(\mathscr{P}, \mathscr{L})$ is an incidence geometry of points and lines satisfying:

(GQ1) Two points are incident with at most one line.

(GQ2) For a non-incident point line pair $(P, m)$ there is a unique point of $m$ collinear with $P$.

(GQ3) No point is collinear with all others.

The dual structure of a GQ (that is, swapping the labels of "points" and "lines") is also a GQ. If a GQ has a line incident with a finite number of points then all lines are incident with exactly this number of points, and dually for lines. A finite GQ has an $\operatorname{order}(s, t)$ for $s, t$ finite such that there are $s+1$ points incident with a line and $t+1$ lines incident with a point. For a comprehensive introduction to finite GQs see [19].

Let $\mathscr{S}=(\mathscr{P}, \mathscr{L})$ be a GQ of order $(s, t)$. Given two (not necessarily distinct) points $X, X^{\prime}$ of $\mathscr{S}$, we write $X \sim X^{\prime}$ and say that $X$ and $X^{\prime}$ are collinear, provided there is some line $m$ incident with both $X$ and $X^{\prime}$. If $X \neq X^{\prime}$ then the line $m$ is unique and we will often use $\left\langle X, X^{\prime}\right\rangle$ to denote the line $m$. For $X \in \mathscr{P}$, put 
$X^{\perp}=\left\{X^{\prime} \in \mathscr{P}: X \sim X^{\prime}\right\}$. If $A \subset \mathscr{P}$, then we define $A^{\perp}=\cap\left\{X^{\perp}: X \in A\right\}$ and $A^{\perp \perp}=\left(A^{\perp}\right)^{\perp}$.

If $s^{2}=t>1$ then by a result of Bose and Shrikhande ([4]) $\left|\{X, Y, Z\}^{\perp}\right|=s+1$ for any triple $\{X, Y, Z\}$ of pairwise non-collinear points (called a triad). Consequently, $\left|\{X, Y, Z\}^{\perp \perp}\right| \leq s+1$ and we say that $\{X, Y, Z\}$ is 3-regular provided $\left|\{X, Y, Z\}^{\perp \perp}\right|=s+1$. The point $X$ is 3-regular if and only if each triad $\{X, Y, Z\}$ is 3-regular.

GQs are exactly the rank 2 polar spaces and so as examples we have the nonsingular quadrics and Hermitian varieties in finite projective spaces that contain lines but no higher dimensional subspaces.

The first non-classical construction of GQs comes from Tits (see [10]). Let $\mathscr{O}$ be an ovoid in $H=\operatorname{PG}(n, q), n=2,3$, embedded in $\Sigma=\mathrm{PG}(n+1, q)$. Then the following structure $T_{n}(\mathscr{O})$ is a GQ of order $\left(q, q^{n-1}\right)$. Points are of three types: (i) the points of $\Sigma \backslash H$; (ii) the $n$-dimensional subspaces of $\Sigma$ meeting $H$ in a tangent space to $\mathscr{O}$; and (iii) a formal point ( $\infty$ ). Lines are of two types: (a) lines of $\Sigma$, not in $H$, meeting $H$ in a point of $\mathscr{O}$; and (b) the points of $\mathscr{O}$. Incidence is natural plus $(\infty)$ is incident with all lines of type (b).

Geometrically, we can see a link between this construction of Tits and Laguerre geometries if we consider dualising the Tits construction in $\mathrm{PG}(n+1, q)$. The important observation that makes this useful is that if $(n, q) \neq\left(2,2^{h}\right)$ for some $h \geq 1$, then under a duality of $\operatorname{PG}(n, q)$ the points of an ovoid $\mathscr{O}$ of $\operatorname{PG}(n, q)$ are mapped to a set of hyperplanes which are the set of tangent hyperplanes to an ovoid equivalent to $\mathscr{O}$ (see a combination of $[1,14,20-22]$ ). So if $\mathscr{O}$ is an ovoid in $H=\operatorname{PG}(n, q)$, $n=2,3$, embedded in $\Sigma=\operatorname{PG}(n+1, q)$, then dualising $\Sigma$ we have that $\widehat{H}$ is a point, the points of $\mathscr{O}$ become hyperplanes on $\widehat{H}$ that are in fact the hyperplanes obtained by taking the span of $\widehat{H}$ with the tangent planes to an ovoid equivalent to $\mathscr{O}$ in an $n$-dimensional subspace not containing $\widehat{H}$. That is, the points of $\mathscr{O}$ become tangent planes to a cone with an ovoid equivalent to $\mathscr{O}$ as a base, with lines the dual of the tangent planes to $\mathscr{O}$. Hence, if $\mathscr{K}$ is such a cone with vertex $V$, we may represent the construction of Tits above by: a point of type (i) corresponds to an ovoidal hyperplane section of $\mathscr{K}$, a point of type (ii) corresponds to a point of $\mathscr{K} \backslash\{V\},(\infty)$ corresponds to $V$, a line of type (a) corresponds to an $(n-1)$-dimensional subspace contained in a tangent plane to $\mathscr{K}$, but not containing $V$ and finally a line of type (b) corresponds to a hyperplane tangent to $\mathscr{K}$ and hence corresponds to a line of $\mathscr{K}$. Note that if we take a $(n-1)$-dimensional subspace not containing $V$ and contained in a tangent plane to $\mathscr{K}$, the hyperplanes on this subspace intersect $\mathscr{K}$ in a pencil of the Laguerre geometry $\mathscr{S}$ constructed from $\mathscr{K}$. In this way the above correspondence is actually a correspondence between the geometry of $T_{n}(\mathscr{O})$, removing the point $(\infty)$, and the geometry of $\mathscr{S}$.

The above discussion raises the question: when does a Laguerre geometry give 
rise to a GQ? In the above connection, circles of the Laguerre geometry are points of the GQ and the pencils of circles of the Laguerre geometry correspond to lines of the GQ. So, given the uniqueness in Axiom (GQ2), a Laguerre geometry must at least satisfy:

(GQ) Three pairwise touching circles are incident with a common point (of contact).

Given this Condition we can determine when a Laguerre geometry corresponds to a GQ and conversely. For the following theorem we note that a centre of a triad is a point collinear with all three points of the triad.

THEOREM 3.1. Let $\mathscr{S}=(\mathscr{P}, \mathscr{L}, \mathscr{C})$ be a finite Laguerre geometry with parameters $n, s, \ell$ satisfying Axiom GQ. Consider the incidence structure which has points of three types: (i) points of $\mathscr{S}$; (ii) circles of $\mathscr{S}$; and (iii) a formal point $(\infty)$. The lines are of two types: (a) lines of $\mathscr{S}$; and (b) pencils of circles. Incidence is that inherited from $\mathscr{S}$, plus $(\infty)$ is incident with all lines of type $(a)$ and a point of $\mathscr{S}$ is incident with all pencils for which it is the base point. This incidence structure is a $G Q$, denoted $\mathrm{GQ}(\mathscr{S})$, if and only if $\mathscr{S}$ satisfies $\mathrm{GQ}$ and $\ell=n s+1$, in which case the $G Q$ has order $(n, n s)$.

Conversely, let $\mathscr{S}=(\mathscr{P}, \mathscr{L})$ be a generalized quadrangle of order $(\bar{s}, \bar{t})$ with point $(\infty)$ such that each triad of $\mathscr{S}$ with $(\infty)$ as a centre has exactly $s+1$ centres for some fixed $s$. Then the incidence structure

$\left((\infty)^{\perp} \backslash\{(\infty)\},\{m \in \mathscr{L}:(\infty)\right.$ is incident with $\left.m\},\left\{X^{\perp} \cap(\infty)^{\perp}: X \in \mathscr{P} \backslash(\infty)^{\perp}\right\}\right)$, where incidence is induced by that of $\mathscr{S}$, is a Laguerre geometry with $s=\bar{t} / \bar{s}, n=\bar{s}$, $\ell=\bar{t}+1$ and satisfying $G Q$.

PROOF. Given a Laguerre geometry satisfying GQ, the incidence structure given has the property that given a non-incident line pair $(P, m)$ there is at most one line incident with $P$ and concurrent with $m$. The only case in which this number is possibly less than 1 is where $P$ is a circle of $\mathscr{S}$ and $m$ is a pencil of circles of $\mathscr{S}$ whose base point is not contained in the circle $P$. Counting, we see that there are $\ell(n-1)$ circles touching $P$ and $(n \ell-\ell) n s$ pencils whose base point is not on $P$. Since each circle touching $P$ is contained in $\ell-1$ pencils whose base point is not on $P$, we have a GQ exactly when $\ell(n-1)(\ell-1)=(n \ell-\ell) n s$, that is $\ell=n s+1$.

We can extract two cases of special interest from this theorem.

THEOREM 3.2. Let $\mathscr{S}$ be a Laguerre geometry with parameters $n, s$, $\ell$ such that $\ell=n s+1$.

(1) If $\mathscr{S}$ satisfies $G Q$ then $s+1 \mid n+1$ and hence if $s=1$ then $n$ is odd.

(2) If $n=s$ then $\mathscr{S}$ satisfies $G Q$. 
PROOF. Since $\ell=n s+1$, the possible intersection numbers for circles are $0,1, s+1$. Let $C$ and $C^{\prime}$ be two circles of $\mathscr{S}$ touching at the point $P$. Let $Q$ be any point of $C^{\prime} \backslash\{P\}$, let $R$ be the point of $C$ collinear with $Q$ and consider the circles touching $C^{\prime}$ at $Q$. Each of these $n-1$ circles meets $C \backslash\{P, R\}$ in either 0,1 or $s+1$ points and each point of $C \backslash\{P, R\}$ is contained in a unique such circle. Hence $a(s+1)+b=n s-1$ where $a, b$ are non-negative integers and $a+b \leq n-1$.

If $\mathscr{S}$ satisfies GQ then $b=0$ and we have $s+1 \mid n s-1$ or equivalently $s+1 \mid n+1$.

If $n=s$ then $a(s+1)+b=s^{2}-1$ and $a+b \leq s-1$, from which it follows that $a=s-1, b=0$ and $\mathscr{S}$ satisfies GQ.

REMARK. Note that the converse part of Theorem 3.1 also follows from $[19$, ??? 1.7.11.

What we see from the above theorem is that a Laguerre geometry with $n=s$ and $\ell=n s+1$ always gives rise to a GQ of order $\left(s, s^{2}\right)$. Conversely, every GQ of $\operatorname{order}\left(\bar{s}, \bar{s}^{2}\right)$ has the property that every triad has $\bar{s}+1$ centres ([4]) and so from every point of such a GQ there arises a Laguerre geometry with parameters $n=s=\bar{s}$ and $\ell=\bar{s}^{2}+1$.

There are many examples of GQs of order $\left(s, s^{2}\right)$, for instance the GQs $T_{3}(\mathscr{O})$ for $\mathscr{O}$ an ovoid of $\mathrm{PG}(3, q)$, as mentioned earlier, the dual of GQs arising from the flock of a quadratic cone, as well as certain translation generalized quadrangles (a generalisation of the $T_{3}(\mathscr{O})$ construction). See [26] for more details. So we have many constructions of Laguerre geometries from these GQs and interestingly in most cases the Laguerre geometry in question does not have classical internal structure at any point.

Finally, we mention that $[7,17,18]$ contain other connections between Laguerre planes and generalized quadrangles. Of particular interest are $[17,18]$ in which it is shown (amongst other things) that a GQ of order $(s, s)$ with a distinguished antiregular point is equivalent to a Laguerre plane of odd order $s$.

\section{Laguerre geometries with classical internal structure and GQs satisfying Property (G)}

In Theorem 3.2 we saw that a Laguerre geometry with parameters $n=s$ and $\ell=s^{2}+1$ always gives rise to a GQ of order $\left(s, s^{2}\right)$ via the construction given in Theorem 3.1 and conversely. In this section we investigate Laguerre geometries with these parameters that also have classical internal structure at a point. It turns out that this property has been well studied from the quadrangle point of view in the form of Property $(G)$, which we shall now introduce (see [16]). We note that much of the work in this section is inspired by that in [27] and [5]. 
Let $\mathscr{S}=(\mathscr{P}, \mathscr{L})$ be a GQ of order $\left(s, s^{2}\right)$ with $s \neq 1$. Let $X_{1}, Y_{1}$ be distinct collinear points. We say that the pair $\left\{X_{1}, Y_{1}\right\}$ has Property $(G)$, or that $\mathscr{S}$ has Property $(G)$ at $\left\{X_{1}, Y_{1}\right\}$, if every triad $\left\{X_{1}, X_{2}, X_{3}\right\}$ of points; with $Y_{1} \in\left\{X_{1}, X_{2}, X_{3}\right\}^{\perp}$, is 3-regular. The GQ $\mathscr{S}$ has Property $(G)$ at the line $m$, or the line $m$ has Property $(G)$, if each pair of distinct points incident with $m$ has Property $(\mathrm{G})$. If $(X, m)$ is a flag then we say that $\mathscr{S}$ has Property $(G)$ at $(X, m)$ or that $(X, m)$ has Property $(G)$, if every pair $\{X, Y\}, X \neq Y$ and $Y$ incident with $m$ has Property $(G)$.

Suppose that $\mathscr{S}=(\mathscr{P}, \mathscr{L})$ is a GQ of $\operatorname{order}\left(s, s^{2}\right)$ satisfying Property $(\mathrm{G})$ at the pair of points $\{X, Y\}$. We now review a construction of $\mathrm{AG}(3, s)$ from $\mathscr{S}, X$ and $Y$ due to Payne and Thas (see [25]).

We consider the following incidence structure $\mathscr{S}_{X Y}=\left(\mathscr{P}_{X Y}, \mathscr{L}_{X Y}\right)$ :

(i) $\mathscr{P}_{X Y}=X^{\perp} \backslash\{X, Y\}^{\perp \perp}$.

(ii) Elements of $\mathscr{L}_{X Y}$ are of two types: (a) the sets $\{Y, Z, U\}^{\perp \perp} \backslash\{Y\}$, with $\{Y, Z, U\}$ a triad with $X \in\{Y, Z, U\}^{\perp}$, and (b) the sets $\{X, W\}^{\perp} \backslash\{X\}$, with $X \sim W \nsucc Y$.

(iii) Incidence is given by containment.

Then we have the following result.

THEOREM 4.1 (Payne and Thas, see [25]). The incidence structure $\mathscr{S}_{X Y}$ is the design of points and lines of the affine space $\mathrm{AG}(3, s)$. In particular, $s$ is a prime power $q$.

The planes of the affine space $\mathscr{S}_{X Y}=\mathrm{AG}(3, q)$ are of two types:

(a) The sets $\{X, Z\}^{\perp} \backslash\{Y\}$, with $X \not \mathcal{Z}$ and $Y \in\{X, Z\}^{\perp}$, and

(b) each set which is the union of all elements of type (b) of $\mathscr{L}_{X Y}$ containing a point of some line $m$ of type (a) of $\mathscr{L}_{X Y}$.

This construction leads us to an equivalent formulation of Property $(\mathrm{G})$ at a pair of points (see [2]).

THEOREM 4.2. Let $\mathscr{S}=(\mathscr{P}, \mathscr{L})$ be a $G Q$ of order $\left(s, s^{2}\right)$ and $X, Y \in \mathscr{P}$ with $X \sim Y$. Then $\mathscr{S}$ satisfies Property $(G)$ at $\{X, Y\}$ if and only if the incidence structure

Points: $X^{\perp} \backslash\langle X, Y\rangle$,

Planes: $Y^{\perp} \backslash\langle X, Y\rangle$,

Incidence: Collinearity in $\mathscr{S}$,

is the point-plane incidence structure of $\mathrm{PG}(3, s)$ with an incident point-plane pair removed.

The classical GQ $Q(5, q)$ of order $\left(q, q^{2}\right)$ satisfies Property $(\mathrm{G})$ at every pair of collinear points. If $\Omega$ is an ovoid of $\operatorname{PG}(3, q)$ then the Tits GQ $T_{3}(\Omega)$ of order $\left(q, q^{2}\right)$ (see [10]) satisfies Property (G) at a flag. A flock of a quadratic cone in $\operatorname{PG}(3, q)$ is a 
partition of the non-vertex points of the cone into plane sections (and so may be seen as a partition of the points of the associated Laguerre plane into circles). In [24], Thas showed that to a flock of a quadratic cone there corresponds a GQ of order $\left(q^{2}, q\right)$ (which is often called a flock $G Q$ in the literature) previously constructed via group coset geometry methods by Kantor ([11]) in the $q$ odd case; and Payne ([15]) in the $q$ even case. In [16], Payne showed that the dual of this GQ satisfies Property (G) at a line, for both $q$ odd and even. Note that $Q(5, q)$ may also be thought of as a Tits GQ and a dual flock GQ.

If we consider a GQ $\mathscr{S}$ of order $\left(s, s^{2}\right)$ with a point $X$, then by Theorem 3.1 we can construct a Laguerre geometry $\mathscr{S}_{X}$ from $\mathscr{S}$ and $X$. If $Y$ is any other point of $\mathscr{S}$ collinear with $X$ (and hence also a point of $\mathscr{S}_{X}$ ) then the points and hyperplanes of the internal structure of $\mathscr{S}_{X}$ at $Y$ are exactly the points and "planes" (see Theorem 4.1) of the structure $\mathscr{S}_{X Y}$. Using these connections we have the following result.

THEOREM 4.3. Let $\mathscr{S}=(\mathscr{P}, \mathscr{L})$ be a $G Q$ of order $\left(s, s^{2}\right)$, let $X \in \mathscr{P}$ and let $\mathscr{S}_{X}$ be the Laguerre geometry corresponding to $X$ (as in Theorem 3.1). Then $\mathscr{S}$ satisfies Property $(G)$ at a pair of distinct, collinear points $\{X, Y\}$ if and only if $\mathscr{S}_{X}$ has classical internal structure at the point $Y$.

Now suppose that $\mathscr{S}$ is a GQ of order $\left(q, q^{2}\right)$ satisfying Property $(\mathrm{G})$ at the pair of collinear points $\{X, Y\}$. Let $\overline{\mathscr{S}}_{X Y}$ be the projective completion of $\mathscr{S}_{X Y}$ with plane at infinity $\pi_{\infty}$. In [27], Thas gives the following interpretation of the GQ $\mathscr{S}$ in $\overline{\mathscr{S}_{X Y}}$. The $q^{2}$ lines of type (b) of $\mathscr{S}_{X Y}$ are parallel, so they define a point $\infty$ of $\overline{\mathscr{S}}_{X Y}$. If we now consider any $Z \in \mathscr{P}$ with $X \nsim Z \nsucc Y$ and $U$ the point of $\langle X, Y\rangle$ such that $Z \sim U$, then $\mathscr{V}=\{X, Z\}^{\perp} \backslash\{U\}$ is a set of $q^{2}$ points. Clearly each line of $\overline{\mathscr{S}_{X Y}}$ on $\infty$ meets $\mathscr{V}$ in exactly one point. Further, if $U_{1}, U_{2}, U_{3}$ are points of $\mathscr{V}$ collinear in $\overline{\mathscr{S}_{X Y}}$ then it must be that $Y \in\left\{U_{1}, U_{2}, U_{3}\right\}^{\perp \perp}$ and so $Z \sim Y$, a contradiction since $X, Y, Z$ is a triangle. It follows from this that $\mathscr{V} \cup\{\infty\}$ is an ovoid of $\overline{\mathscr{S}_{X Y}}$ with tangent plane $\pi_{\infty}$ at $\infty$. We will denote this ovoid by $\Omega_{z}$.

Thas also determined the intersections of these ovoids. Consider two distinct points $Z_{1}, Z_{2} \in \mathscr{P} \backslash X^{\perp}$ with $Z_{1}, Z_{2}$ collinear with points $U_{1}, U_{2}$ on $\langle X, Y\rangle$, respectively, with $U_{1}, U_{2} \neq X, Y$. If $Z_{1} \sim Z_{2}$ and $U_{1}=U_{2}$ then $\Omega_{Z_{1}} \cap \Omega_{Z_{2}}=\{\infty\}$, since any larger intersection yields a triangle in $\mathscr{S}$.

If $Z_{1} \sim Z_{2}$ and $U_{1} \neq U_{2}$ then $\Omega_{Z_{1}} \cap \Omega_{Z_{2}}=\{\infty, R\}$ where $R$ is the point of the line $\left\langle Z_{1}, Z_{2}\right\rangle$ in $X^{\perp}$. Further, the point of $\left\langle Z_{1}, Z_{2}\right\rangle$ in $Y^{\perp}$ corresponds, in $\overline{\mathscr{S}_{X Y}}$, to a plane which is tangent at $R$ to both $\Omega_{Z_{1}}$ and $\Omega_{Z_{2}}$.

If $Z_{1} \nsucc Z_{2}$ and $U_{1}=U_{2}$ then $\Omega_{Z_{1}} \cap \Omega_{Z_{2}}=\left(\left\{X, Z_{1}, Z_{2}\right\}^{\perp} \backslash\left\{U_{1}\right\}\right) \cup\{\infty\}$, an intersection of size $q+1$.

For the last case, if $Z_{1} \ngtr Z_{2}$ and $U_{1} \neq U_{2}$ then $\Omega_{Z_{1}} \cap \Omega_{Z_{2}}=\left\{X, Z_{1}, Z_{2}\right\}^{\perp} \cup\{\infty\}$, an intersection of size $q+2$.

If $m$ is a line of $\mathscr{S}$ such that $m$ and $\langle X, Y\rangle$ are non-concurrent then let the set of 
ovoids of PG $(3, q)=\overline{\mathscr{S}_{X Y}}$ corresponding to points of $m \backslash\left(X^{\perp} \cup Y^{\perp}\right)$ be denoted $\mathscr{T}$. The set $\mathscr{T}$ is a set of $q-1$ ovoids of $\operatorname{PG}(3, q)$ meeting pairwise in exactly two fixed points and sharing the tangent planes at those two fixed points. We will call such a set $\mathscr{T}$ a transversal of ovoids. These two common points are called the base points of the transversal and the two common tangent planes are called the base planes of the transversal. In the Laguerre geometry $\mathscr{S}_{X}$ the transversal of ovoids $\mathscr{T}$ corresponds to the circles not incident with $Y$ in a pencil and the base plane of $\mathscr{T}$ that is not $\pi_{\infty}$ corresponds to the circle in the pencil incident with $Y$.

In $[2,5]$ the set of ovoids corresponding to a GQ of order $\left(q, q^{2}\right)$ satisfying Property $(G)$ at a pair of collinear points was studied and characterised under a certain hypothesis. In particular, we have the following result.

THEOREM 4.4 ([2]). Let $\mathscr{S}$ be a $G Q$ of order $\left(q, q^{2}\right), q$ odd, satisfying Property $(G)$ at a pair of points. Then $\mathscr{S}$ is the dual of a flock $G Q$.

Consequently, in the $q$ odd case we know that a Laguerre geometry with classical internal structure at a point arises from a dual flock GQ.

Since the $q$ odd case is settled, we now turn our attention to the $q$ even case. By Theorem 4.3 and Theorem 3.1, we know that the set of ovoids of $\operatorname{PG}(3, q)$ associated with a GQ $\mathscr{S}$ of order $\left(q, q^{2}\right)$ satisfying Property $(\mathrm{G})$ at a pair $\{X, Y\}$ may be used to construct a Laguerre geometry with classical internal structure at a point. In fact, it can be shown that this property characterises the set of ovoids. That is, if we take a set of ovoids with a common point and common tangent plane at that point and can construct a Laguerre geometry with classical internal structure at a point then we can reconstruct a GQ with Property (G) at a pair of points. Further, since we insist that the Laguerre geometry constructed from the set of ovoids has classical internal structure at a point it turns out that the Axiom (L2) (the touching axiom) of the Laguerre geometry is implied by Axioms (L1*), (L3), (L4) and the classical internal structure at a point. This allows us to prove a stronger characterisation of the set of ovoids by omitting the condition equivalent to Axiom (L2) of a Laguerre geometry. We now work towards proving this stronger characterisation. In what follows a 4-cap of a projective space is a set of four points no three of which are collinear.

Let $\left(\infty, \pi_{\infty}\right)$ be an incident point-plane pair of $\operatorname{PG}(3, q)$. Then a Laguerre set $\Theta$ of ovoids of $\mathrm{PG}(3, q)$ with respect to $\left(\infty, \pi_{\infty}\right)$ is a set of ovoids each of which contains $\infty$ and has tangent plane $\pi_{\infty}$ such that if $X, Y, Z$ are three distinct points of $\mathrm{PG}(3, q) \backslash \pi_{\infty}$ with $\{\infty, X, Y, Z\}$ a 4-cap in $\mathrm{PG}(3, q)$, then:

(A1) if $\infty \in\langle X, Y, Z\rangle$ then $|\{\Omega \in \Theta:\{X, Y, Z\} \subset \Omega\}|=q$; and

(A2) if $\infty \notin\langle X, Y, Z\rangle$ then $|\{\Omega \in \Theta:\{X, Y, Z\} \subset \Omega\}|=q-1$.

Our aim is to show that we can construct a Laguerre geometry with classical internal structure at a point from a Laguerre set. 
LEMMA 4.5. $|\Theta|=q^{3}(q-1)$.

PROOF. There are $\left(q^{2}+q\right) q^{2}\left(q^{2}-q\right)\left(q^{2}-3 q+2\right)$ ordered triples $(X, Y, Z)$ of points in $\mathrm{PG}(3, q) \backslash \pi_{\infty}$ such that $\infty \in\langle X, Y, Z\rangle=\pi$ and $\{\infty, X, Y, Z\}$ is a 4-cap in $\pi$. For an ovoid $\Omega$ with tangent plane $\infty$ at $\pi_{\infty}$, there are $\left(q^{2}+q\right) q(q-1)(q-2)$ such ordered triples contained in $\Omega$. Hence $|\Theta|=q^{3}(q-1)$.

LEMMA 4.6. Let $X \in \mathrm{PG}(3, q) \backslash \pi_{\infty}$. Then $X$ is contained in $q^{2}(q-1)$ elements of $\Theta$.

PROOF. Let $\pi$ be a plane on the line $m=\langle\infty, X\rangle$. There are $\left(q^{2}-q\right)\left(q^{2}-3 q+2\right)$ triples $(X, Y, Z)$ of points in $\pi \backslash \pi_{\infty}$ such that $\{\infty, X, Y, Z\}$ is a 4-cap. Thus there are $(q+1)\left(q^{2}-q\right)\left(q^{2}-3 q+2\right)$ triples considering all planes on $m$. By (A1), there are $q(q+1)\left(q^{2}-q\right)\left(q^{2}-3 q+2\right)$ quadruples $(X, Y, Z, \Omega)$ with $\Omega \in \Theta$ containing $X, Y, Z$. However, each element of $\Theta$ containing $X$ also contains $(q+1)(q-1)(q-2)$ triples $(X, Y, Z)$ with $\infty \in\langle X, Y, Z\rangle$. Hence the number of elements of $\Theta$ containing $X$ is $q^{2}(q-1)$.

LEMma 4.7. Let $X, Y \in \mathrm{PG}(3, q) \backslash \pi_{\infty}$ be such that $\infty \notin\langle X, Y\rangle$. Then there are exactly $q^{2}-q$ elements of $\Theta$ containing $X$ and $Y$.

PROOF. Any element of $\Theta$ containing $X$ and $Y$ meets $\pi=\langle\infty, X, Y\rangle$ in an oval and hence there are $q-2$ triples $(X, Y, Z)$, with $Z \in \pi$, contained in such an ovoid. In total there are $q^{2}-3 q+2$ triples $(X, Y, Z)$ in $\pi \backslash \pi_{\infty}$ such that $\{\infty, X, Y, Z\}$ is a 4-cap. Hence there are $q^{2}-q$ elements of $\Theta$ containing $X$ and $Y$.

Lemma 4.8. Let $X \in \mathrm{PG}(3, q) \backslash \pi_{\infty}$ and $\pi$ be a plane such that $X \in \pi$ but $\infty \notin \pi$. Then there are exactly $q-1$ elements of $\Theta$ containing $X$ and with tangent plane $\pi$ at $X$.

PROOF. We count how many elements of $\Theta$ contain $X$ with $\pi$ not a tangent plane, that is, meeting $\pi$ in an oval. There are $q^{2}-1$ choices for a point $Y \in \pi \backslash\left(\pi_{\infty} \cup\{X\}\right)$ and hence $\left(q^{2}-q\right)\left(q^{2}-1\right)$ triples $(X, Y, \Omega)$ with $\Omega \in \Theta$ and containing $X$ and $Y$. Now for each element of $\Theta$ containing $X$ and with $\pi$ not a tangent plane there are $q$ choices for $Y$ and so the number of elements of $\Theta$ containing $X$ and with $\pi$ not tangent is $(q-1)\left(q^{2}-1\right)$. Hence the number of elements of $\Theta$ containing $X$ and with $\pi$ a tangent plane is $q^{2}(q-1)-(q-1)\left(q^{2}-1\right)=q-1$.

Lemma 4.9. Let $X \in \mathrm{PG}(3, q) \backslash \pi_{\infty}$ and $\pi$ be a plane such that $X \in \pi$ but $\infty \notin \pi$. Then there are exactly $(q-1)^{2}$ elements $\Omega$ of $\Theta$ whose associated polarity interchanges $X$ and $\pi$ and such that $X \notin \Omega$. 
PROOF. If $\Omega \in \Theta$ then we let $\perp_{\Omega}$ denote the symplectic polarity defined by $\Omega$. We count the number of cases where $X \notin \Omega \in \Theta$ and $X^{L_{\Omega}} \neq \pi$. There are $(q+1)(q-1)(q-2)$ ordered collinear triples $(X, Y, Z)$ in $\pi \backslash \pi_{\infty}$ and $q^{2}-q$ elements of $\Theta$ on $Y$ and $Z$ (and hence not on $X$ ). For $\Omega \in \Theta$ not containing $X$ and with $X^{\perp_{\Omega}} \neq \pi$ there are $q$ such ordered pairs $(Y, Z)$. Hence the number of $\Omega \in \Theta$ such that $X \notin \Omega$ and $X^{\perp_{n}} \neq \pi$ is $(q+1)(q-1)^{2}(q-2)$. By Lemma 4.8, the number of $\Omega \in \Theta$ such that $X \notin \Omega, X^{\perp_{\Omega}} \neq \pi$ and $\pi$ is a tangent plane to $\Omega$ is $\left(q^{2}-1\right)(q-1)$. Hence the number of $X \notin \Omega$ for which $X^{\perp \Omega}=\pi$ is $\left(q^{3}(q-1)-q^{2}(q-1)\right)-(q+1)(q-1)^{2}(q-2)-(q-1)^{2}(q+1)=(q-1)^{2}$.

LEMMA 4.10. Let $X \in \mathrm{PG}(3, q) \backslash \pi_{\infty}$ and $\pi$ be a plane such that $X \in \pi$ but $\infty \notin \pi$. There are exactly $q(q-1)$ elements of $\Theta$ with associated polarity that interchanges $X$ and $\pi$.

PROOF. This follows from the previous two lemmas.

LEMMA 4.11. Let $X \in \pi_{\infty}, X \neq \infty$, and let $\pi$ be a plane distinct from $\pi_{\infty}$ such that $X, \infty \in \pi$. There are exactly $q^{2}(q-1)$ elements of $\Theta$ with associated polarity interchanging $X$ and $\pi$.

PROOF. There are $q^{2}(q-1)$ ordered pairs of points in $\pi \backslash \pi_{x}$ whose span contains $X$ and each such pair is contained in $q^{2}-q$ elements of $\Theta$ by Lemma 4.7. Each such element of $\Theta$ contains $q$ such ordered pairs and so there are $q^{2}(q-1)^{2}$ elements of $\Theta$ whose polarity does not interchange $X$ and $\pi$. Hence there are $q^{2}(q-1)$ elements of $\Theta$ whose polarity does interchange $X$ and $\pi$.

In [5], an equivalence relation is constructed amongst the symplectic polarities that interchange $\infty$ and $\pi_{\infty}$. Two such polarities $\perp_{1}$ and $\perp_{2}$ are equivalent if and only if $\perp_{1} \circ \perp_{2}$ acts as the identity on some line $m$ incident with $\infty$ and contained in $\pi_{x}$. The two polarities are said to be equivalent about $m$.

If $\phi_{1}$ and $\phi_{2}$ are inequivalent polarities then there exist skew lines $m_{1}, m_{2}$ with $\infty \in m_{1} \not \subset \pi_{\infty}$ and $\infty \notin m_{2} \subset \pi_{\infty}$ such that $\phi_{1} \circ \phi_{2}$ fixes the points and planes incident with $m_{1}$ and $m_{2}$, and $m_{1}^{\phi_{1}}=m_{1}^{\phi_{2}}=m_{2}$. The polarities are said to be inequivalent about $\left\{m_{1}, m_{2}\right\}$.

We will also extend this equivalence relation to the elements of $\Theta$ and talk of ovoids being equivalent (about a line) or inequivalent (about a pair of skew lines).

LEMMA 4.12. Amongst the symplectic polarities of $\mathrm{PG}(3, q)$ interchanging $\infty$ and $\pi_{\infty}$, two equivalent polarities are induced by the same number of elements of $\Theta$. Furthermore, the number of elements of $\Theta$ with induced polarity in a fixed class is a multiple of $q^{2}$. 
PROOF. For some fixed symplectic polarity $\perp$ with $\infty^{\perp}=\pi_{\infty}$, define

$a=$ number of elements of $\Theta$ inducing $\perp$,

$b=$ number of elements of $\Theta$ inducing a polarity distinct and equivalent to $\perp$,

$c=$ number of elements of $\Theta$ inducing a polarity inequivalent to $\perp$.

\section{Hence}

$$
a+b+c=q^{3}(q-1)
$$

Now we count pairs $(X, \Omega)$ where $X \in \operatorname{PG}(3, q) \backslash \pi_{\infty}, \Omega \in \Theta$ and if $\perp_{\Omega}$ is the polarity defined by $\Omega$ then $X^{\perp}=X^{\perp_{2}}$. This count is $q^{3}(q-1) q=q^{4}(q-1)$ by Lemma 4.10.

On the other hand, if $X^{\perp}=X^{\perp_{\Omega}}$ then, since $X \notin \pi_{\infty}$, either $\perp_{\Omega}=\perp$ or $\perp$ and $\perp_{\Omega}$ are inequivalent. If $\perp_{\Omega}=\perp$ then $X^{\perp}=X^{\perp_{\Omega}}$ for all $X \in \operatorname{PG}(3, q) \backslash \pi_{\infty}$, adding $q^{3} a$ to the count. If $\perp$ and $\perp_{\Omega}$ are inequivalent, then there are exactly $q$ points $X$ (on a line containing $\infty$ ) such that $X^{\perp}=X^{\perp_{\Omega}}$, adding $c q$ to the count. Hence $q^{4}(q-1)=q^{3} a+c q$, or

$$
q^{2} a+c=q^{3}(q-1) .
$$

From (4.1) and (4.2) we have $b=\left(q^{2}-1\right) a$. Since $a$ is determined by $c$, the number of elements of $\Theta$ inducing a polarity inequivalent to $\perp$, it follows that $a$ is independent of which polarity in the equivalence class of $\perp$ we use.

Also, the number of elements of $\Theta$ inducing a polarity in the class of $\perp$ is $a+b=q^{2} a$.

REMARK. Counting pairs $(X, \Omega)$, where $\Omega \in \Theta, X \in \pi_{\infty} \backslash\{\infty\}$ and $X^{\perp}=X^{\perp_{\Omega}}$, yields the equation $\left(q^{2}+q\right) a+q b+(q+1) c=\left(q^{2}+q\right) q^{2}(q-1)$, however this is not independent of equations (4.1) and (4.2), and so cannot be used to extend Lemma 4.12.

We note that thus far we have used only Condition (A1) of our definition of $\Theta$.

THEOREM 4.13. Let $X \in \mathrm{PG}(3, q) \backslash \pi_{\infty}$ and $\pi$ be a plane such that $X \in \pi$ and $\infty \notin \pi$. Then the $q-1$ elements of $\Theta$ containing $X$ and with tangent plane $\pi$ form a transversal, that is, intersect pairwise in $\{\infty, X\}$ and (hence) partition the points of $\mathrm{PG}(3, q) \backslash\left(\pi_{\infty} \cup \pi \cup(\infty, X)\right)$. Furthermore, the $q-1$ ovoids are pairwise inequivalent about the lines $\pi \cap \pi_{\infty}$ and $(X, \infty)$.

PROOF. We show that if $Y \in \operatorname{PG}(3, q) \backslash\left(\pi_{\infty} \cup \pi \cup\langle\infty, X\rangle\right)$, then there is exactly one element of $\Theta$ containing $X$ with tangent plane $\pi$ that also contains $Y$. 
Let $\pi^{\prime}=\langle\infty, X, Y\rangle$ and $m=\pi \cap \pi^{\prime}$. First count the number of elements of $\Theta$ containing $X, Y$ with $m$ a tangent. In total there are $q(q-1)$ elements of $\Theta$ containing $X, Y$ and to have $m$ not a tangent the ovoid must contain some point $Z \in m \backslash\left(X \cup \pi_{\infty} \cup\langle\infty, Y\rangle\right)$. By (Al) this gives $q(q-2)$ ovoids with $m$ not tangent and hence $q$ elements of $\Theta$ containing $X, Y$ with $m$ tangent.

The number of pairs $(Z, \Omega)$, where $Z \in \pi \backslash\left(\pi_{\infty} \cup m\right)$ and $X, Y, Z \in \Omega \in \Theta$ and $m$ is not tangent to $\Omega$, is $(q-1) q(q-2)$. By (A2), the number of pairs $(Z, \Omega)$ with $Z \in \pi \backslash\left(\pi_{\infty} \cup m\right)$ and $X, Y, Z \in \Omega \in \Theta$ is $\left(q^{2}-q\right)(q-1)$. It thus follows that the number of pairs $(Z, \Omega)$ with $Z \in \pi \backslash\left(\pi_{\infty} \cup m\right), X, Y, Z \in \Omega \in \Theta$ and $m$ a tangent to $\Omega$ is $q(q-1)$. Hence the number of elements of $\Omega \in \Theta$ with $X, Y \in \Omega, m$ tangent to $\Omega$ and $\pi$ not a tangent plane is $q-1$, from which it follows that there is exactly one element of $\Theta$ containing $X$ and $Y$ and with tangent plane $\pi$.

So the $q-1$ elements of $\Theta$ containing $X$ and with tangent plane $\pi$ must partition the points of $\mathrm{PG}(3, q) \backslash\left(\pi_{\infty} \cup \pi \cup\langle\infty, X\rangle\right)$ and hence form a transversal.

Let the transversal be $\left\{\Omega_{1}, \Omega_{2}, \ldots, \Omega_{q-1}\right\}$. Since all ovoids in the transversal have tangent plane $\pi$ at $X$ and tangent plane $\pi_{\infty}$ at $\infty$, it must be that either any two ovoids in the transversal are inequivalent about the skew lines $\pi \cap \pi_{\infty}$ and $\langle\infty, X\rangle$, or they define the same polarity. We show that the latter possibility cannot occur. Consider a plane $\pi^{\prime}$ such that $\infty \in \pi^{\prime}$ and $X \notin \pi^{\prime}$. Let $P=\pi^{\prime} \cap \pi \cap \pi_{x}$. Then $\Omega_{i} \cap \pi^{\prime}=\mathscr{O}_{i}$ is an oval. Now consider any line $m$ of $\pi^{\prime}$ such that $P, \infty \notin m$. The $q-1$ points of $m \backslash\left(\pi \cup \pi_{\infty}\right)$ are partitioned by the $\mathscr{O}_{i}$. In particular, since $q-1$ is odd it must be that $m$ is tangent to at least one of the $\mathscr{O}_{i}$ and the point $m \cap \pi_{x}$ is the nucleus of this oval. Hence the nuclei of the $\mathscr{O}_{i}$ are the $q-1$ distinct points of $\pi^{\prime} \cap \pi_{x} \backslash\{\infty, P\}$. Hence the polarities of the $\Omega_{i}$ are pairwise distinct.

COROLLARY 4.14. Each symplectic polarity of $\mathrm{PG}(3, q)$ interchanging $\infty$ and $\pi_{x}$ is induced by q elements of $\Theta$. Further, each equivalence class of $\Theta$ contains exactly $q^{3}$ elements.

Proof. Let $X \in \operatorname{PG}(3, q) \backslash \pi_{\infty}$ and let $\pi$ be a plane incident with $X$ but not $\infty$. Then the elements of $\Theta$ with tangent plane $\pi$ at $X$ form a transversal and there is one ovoid from each equivalence class. Considering all such $X$ and $\pi$, we see that the number of elements of $\Theta$ in a given equivalence class is $q^{3} q^{2} / q^{2}=q^{3}$. Using the equations in the proof of Lemma 4.12, we have that $a+b=q^{3}$ and hence $a=q$.

We now have enough information to construct a Laguerre geometry from a Laguerre set.

THEOREM 4.15. Let $\Theta$ be a Laguerre set of ovoids of $\mathrm{PG}(3, q)$, q even, with respect to the incident point-plane pair $\left(\infty, \pi_{\infty}\right)$. Let $\mathscr{S}$ be the incidence structure with points of three types: (i) points of $\mathrm{PG}(3, q) \backslash \pi_{\infty}$; (ii) equivalence classes of $\Theta$; and (iii) $\pi_{\infty}$. 
The lines are of two types: (a) the lines of $\mathrm{PG}(3, q) \backslash \pi_{\infty}$ incident with $\infty$; and $(b)$ a formal line $[\infty]$. The circles are of two types: $(\alpha)$ the elements of $\Theta$; and $(\beta)$ the planes of $\operatorname{PG}(3, q)$ not incident with $\infty$. Incidence is natural, plus the line $[\infty]$ is incident with all points of type (ii) and (iii), while $\pi_{\infty}$ is incident with all circles of type $(\beta)$. Then $\mathscr{S}$ is a Laguerre geometry with parameters $n=s=q, \ell=q^{2}+1$ and with classical internal structure at the point $\pi_{\infty}$.

PROOF. Axioms (A1) and (A2) of a Laguerre set ensure that three non-collinear points of $\mathscr{S}$ of type (i) are incident with $q$ common circles. Two non-collinear points of type (i) and $\pi_{\infty}$ are incident with $q$ common circles of type $(\beta)$. If $X, Y$ are two distinct non-collinear points of type (i) then let $\pi$ be any plane with $X, Y \in \pi$ and $\infty \notin \pi$ and let $P=\langle X, Y\rangle \cap \pi_{\infty}$. For $N \in\left(\pi \cap \pi_{\infty}\right) \backslash\{P\}$, choosing $Z \in\langle X, N\rangle \backslash\{X, N\}$ we have $q-1$ elements of $\Theta$ containing $\{X, Y, Z\}$ by (A2). Hence in total there are $(q-1)^{2}$ elements of $\Theta$ whose intersection with $\pi$ does not have nucleus $N$. Thus by Lemma 4.7 there are exactly $q-1$ elements of $\Theta$ whose intersection with $\pi$ does have nucleus $N$. These ovoids are hence pairwise inequivalent. Fixing any one of these ovoids, we see that for each such plane $\pi$ the ovoid is inequivalent to $q-2$ distinct elements of $\Theta$ that contain $X, Y$ and hence is equivalent to at most $q(q-1)-q(q-2)=q$. Since there are $q(q-1)$ elements of $\Theta$ containing $X, Y$ and $q-1$ equivalence classes, this means there are exactly $q$ in each equivalence class. Hence in $\mathscr{S}$ the points $X, Y$ and a point of type (ii) are incident with exactly $q$ circles. Hence $s=q$ for $\mathscr{S}$.

Theorem 4.13 demonstrates the touching axiom for $\mathscr{S}$ except in the case of touching at a point of type (ii) or (iii), the touching axiom for the latter case being straightfoward to prove. For the final case, first note that by Corollary 4.14 a fixed polarity interchanging $\infty$ and $\pi_{\infty}$ is induced by $q$ elements of $\Theta$, say $\Omega_{1}, \ldots, \Omega_{q}$. Without loss of generality, suppose that $X$ is an element of $\left(\Omega_{1} \cap \Omega_{2}\right) \backslash\{\infty\}$. Then $\Omega_{1}$ and $\Omega_{2}$ have the same tangent plane at $X$ and so define inequivalent polarities by Theorem 4.13, a contradiction. Hence $\Omega_{1}, \ldots, \Omega_{q}$ intersect pairwise in $\infty$ and as circles in $\mathscr{S}$ intersect pairwise in the equivalence class of their polarity. Now we show that no other element of $\Theta$ defining a polarity equivalent to that of $\Omega_{1}$ intersects $\Omega_{1}$ in exactly $\infty$. For the $q^{3}-q$ elements of $\Theta$ equivalent to $\Omega_{1}$ and defining a different polarity to that of $\Omega_{1}$, let $t_{1}, \ldots, t_{q^{3}-q}$ be the intersection sizes of these ovoids with $\Omega_{1} \backslash\{\infty\}$. Then we have $\sum_{i=1}^{q^{3}-q} t_{i}=q^{2}\left(q^{2}-1\right)$ and $\sum_{i=1}^{q^{3}-q} t_{i}\left(t_{i}-1\right)=q^{2}\left(q^{2}-1\right)(q-1)$ and the average value of the $t_{i}$ is $\bar{t}=q$. It follows by calculation that $\sum_{i=1}^{q^{3}-q}\left(t_{i}-\bar{t}\right)^{2}=0$ and hence $t_{j}=\bar{t}$ for $i=1,2, \ldots, q^{3}-q$. So the only elements of $\Theta$ equivalent to $\Omega_{1}$ that intersect $\Omega_{1}$ in exactly $\infty$ are $\Omega_{2}, \ldots, \Omega_{q}$. Hence we have the touching axiom for $\mathscr{S}$.

Finally, the internal structure of $\mathscr{S}$ at $\pi_{\infty}$ is the points and hyperplanes of $\operatorname{PG}(3, q)$ with $\left(\infty, \pi_{\infty}\right)$ removed.

Note that we also have the converse of this theorem. That is, given a Laguerre 
geometry with parameters $n=s=q, \ell=q^{2}+1$ and with classical internal structure at a point, we can construct a GQ of order $\left(q, q^{2}\right)$ satisfying Property $(\mathrm{G})$ at a pair of points whose associated set of ovoids may be readily shown to be a Laguerre set.

\section{Appendix: Elation Laguerre geometries}

Following the theory of Laguerre planes we introduce the idea of an elation Laguerre geometry which is a Laguerre geometry which admits a group acting regularly on the circles, while fixing every line.

Steinke and Löwen $[12,23]$ construct elation Laguerre planes and here we generalise their approach for Laguerre geometries. First, however, we need to introduce the idea of an $O(n, m, q)$-set (see [19]). In $\mathrm{PG}(2 n+m-1, q)$ an $O(n, m, q)$-set is a set of $q^{m}+1(n-1)$-dimensional subspaces

$$
\mathrm{PG}^{(0)}(n-1, q), \mathrm{PG}^{(1)}(n-1, q), \ldots, \mathrm{PG}^{\left(q^{(n)}\right)}(n-1, q),
$$

every three of which generate a PG $(3 n-1, q)$ and such that each element $\mathrm{PG}^{(i)}(n-1, q)$ of $O(n, m, q)$ is contained in a $\mathrm{PG}^{(i)}(n+m-1, q)$ having no point in common with any $\mathrm{PG}^{(j)}(n-1, q)$ for $j \neq i$. An $O(1,1, q)$-set is an oval in $\mathrm{PG}(2, q)$, an $O(1,2, q)$ set is an ovoid of PG(3.q) and more generally, examples of $O(n, n, q)$-sets and $O(n, 2 n, q)$-sets, respectively, are ovals and ovoids over $\operatorname{GF}\left(q^{n}\right)$ "blown up" over the $\mathrm{GF}(q)$ subfield. In [19] it is shown that a $O(n, m, q)$-set gives rise to a translation generalized quadrangle of order $\left(q^{n}, q^{m}\right)$ and conversely.

THEOREM 4.16. Let

$$
\mathrm{PG}^{(0)}(n-1, q), \mathrm{PG}^{(1)}(n-1, q), \ldots, \mathrm{PG}^{\left(q^{m}\right)}(n-1, q)
$$

be an $O(n, m, q)$-set and let

$$
\mathrm{PG}^{(0)}(n+m-1, q)^{*}, \mathrm{PG}^{(1)}(n+m-1, q)^{*}, \ldots, \mathrm{PG}^{\left(q^{m}\right)}(n+m-1, q)^{*}
$$

be the corresponding dual subspaces in $\mathrm{PG}(2 n+m-1, q)$.

Embed $\mathrm{PG}(2 n+m-1, q)$ in $\mathrm{PG}(2 n+m, q)$ and consider the following incidence structure $\mathscr{S}$ of points, lines and circles. Points are the $(n+m)$-dimensional subspaces of $\mathrm{PG}(2 n+m, q)$ meeting $\mathrm{PG}(2 n+m-1, q)$ in a $\mathrm{PG}^{(i)}(n+m-1, q)^{*}$; lines of $\mathscr{S}$ are the subspaces $\mathrm{PG}^{(i)}(n+m-1, q)^{*}, i=0, \ldots, q^{m}$; and circles are the points of $\mathrm{PG}(2 n+m, q) \backslash \mathrm{PG}(2 n+m-1, q)$. Then with the natural incidence $\mathscr{S}$ is an elation Laguerre geometry with $q^{n}$ points incident with each line, $s=q^{m-n}$ and $\ell=q^{m}+1$.

PROOF. Three non-collinear points of $\mathscr{S}$ are three $(n+m)$-dimensional subspaces of $\mathrm{PG}(2 n+m, q)$ intersecting $\mathrm{PG}(2 n+m-1, q)$ in distinct elements of the dual 
of the $O(n, m, q)$-set: Hence they intersect in an $(m-n)$-dimensional subspace of $\operatorname{PG}(2 n+m, q)$ that contains $q^{m-n}$ points of PG $(2 n+m, q) \backslash \mathrm{PG}(2 n+m-1, q)$ and hence for $\mathscr{S}$ we have $s=q^{m-n}$ and it is straightforward to show that $\mathscr{S}$ is a Laguerre geometry. The group of elations with axis $\mathrm{PG}(2 n+m-1, q)$ (that is, the translations of the affine space $\mathrm{PG}(2 n+m, q) \backslash \mathrm{PG}(2 n+m-1, q))$ induce a group of automorphisms on $\mathscr{S}$ acting regularly on the circles, while fixing every line.

Note that for this Laguerre geometry we have that $\ell=n s+1$ (using the notation of Section 2) and it can be shown that if we are not dealing with a $O\left(n, n, 2^{h}\right)$-set, then the tangent spaces form a dual $O(n, m, q)$-set (see [19, Chapter 8] for details). It follows that the Axiom GQ is satisfied in these cases. The corresponding GQ constructed, as in Theorem 3.1, is the translation GQ constructed from the $O(n, m, q)$-set (see [19]). By dualising in $\mathrm{PG}(2 n+m, q)$ this property also allows a representation of the Laguerre geometry using a cone with point vertex over an $O(n, m, q)$-set.

\section{Acknowledgements}

The author wishes to thank the referees for their thorough reading of the paper and the helpful comments provided.

\section{References}

[1] A. Barlotti. 'Un'estensione del teorema di Segre-Kustaanheimo'. Boll. Unione Mat. Ital. 10 (1955). 96-98.

[2] S. G. Barwick. M. R. Brown and T. Pentila. 'Flock generalized quadrangles and tetradic sets of elliptic quadrics of PG(3, q)'. J. Combin. Theory Ser. A 113 (2006). 273-290.

[3] W. Benz. Vorlesung über Geometrie der Algebren. volume 197 of Die Grundlehren der mathematischen Wissenschaften in Einzeldarstellungen (Springer. Berlin. 1937).

[4] R. C. Bose and S. S. Shrikhande. 'Geometric and pseudo-geometric graphs $\left(q^{2}+1 . q+1 . q\right)$ '. J. Geon. 2 (1972). 74-94.

[5] M. R. Brown. 'Projective ovoids and generalized quadrangles'. Ads: Geom. 7 (2007). 65-81.

[6] F. Buekenhout. 'Le plans de benz: Une approach unifiée des plans de Moebius. Laguerre et Minkowski'.J. Geom. 17 (1981), 61-68.

[7] L. R. A. Casse. J. A. Thas and P. R. Wild. ' $\left(q^{n}+1\right)$-sets of $P G(3 n-1, q)$. generalized quadrangles and Laguerre planes'. Bull. Belg. Math. Soc. Simon Stevin 59 (1985). 21-42.

[8] Y. Chen and G. Kaerlein. 'Eine bemerkung über endliche Laguerre- und Minkowski-Ebenen', Geom. Dedicata 2 (1973). 193-194.

[9] W. Cherowitzo. 'Bill Cherowitzo's hyperoval page'. http://www-math.cudenver.edu/ wcherowi/research/hyperoval/hypero.html.

[10] P. Dembowski, Finite Geometries (Springer. Berlin. 1968).

[11] W. M. Kantor. 'Some generalized quadrangles with parameters $\left(q^{2} . q\right)$ '. Math. Z. 192 (1986). 45-50. 
[12] R. Löwen, 'Topological pseudo-ovals, elation Laguerre planes and elation generalized quadrangles'. Math. Z. 216 (1994), 347-369.

[13] C. M. O'Keefe, 'Ovoids in PG(3, q): a survey', Discrete Math. 151 (1996), 175-188.

[14] G. Panella. 'Caratterizzazione dell quadriche di uno spazio (tridimensionale) lineare sopra un corpo finito', Boll. Unione Mat. Ital. 10 (1955), 507-513.

[15] S. E. Payne, 'A new infinite family of generalized quadrangles', Congr. Numer. 49 (1985), 115-128.

[16] - 'An essay on skew translation generalized quadrangles', Geom. Dedicata 32 (1989), 93118.

[17] S. E. Payne and J. A. Thas, 'Generalized quadrangles with symmetry', Bull. Belg. Math. Soc. Simon Stevin 49 (1975/76), 3-32.

[18] — 'Generalized quadrangles with symmetry. II', Bull. Belg. Math. Soc. Simon Stevin 49 (1975/76), 81-103.

[19] - Finite Generalized Quadrangles, volume 110 of Research Notes in Mathematics (Pitman, Boston, MA, 1984).

[20] B. Segre. 'Sulle ovali nei piani lineari finiti', Atti. Accad. Naz. Lincei. Rendic 17 (1954), 141-142.

[21] - 'Ovals in a finite projective plane', Canad. J. Math. 7 (1955). 414-416.

[22] . 'On complete caps and ovaloids in three-dimensional Galois spaces of characteristic two', Acta Arith. 5 (1959), 282-286.

[23] G. F. Steinke, 'On the structure of finite elation Laguerre planes', J. Geom. 41 (1991). 162-179.

[24] J. A. Thas. 'Generalized quadrangles and flocks of cones', European J. Combin. 8 (1987). $441-452$.

[25] - 'Generalized quadrangles of order $\left(s, s^{2}\right)$ I', J. Combin. Theory Ser. A 67 (1994), 140-160.

[26] —_. 'Generalized polygons', in: Handbook of Incidence Geometry (ed. F. Buenkenhout) (Elsevier. Amsterdam. 1995) chapter 9. 383-431.

[27] — 'Generalized quadrangles of order $\left(s, s^{2}\right)$, III', J. Combin. Theory Ser. A 87 (1999), 247272.

[28] J. Tits. 'Sur le trialité et certains groupes qui s'en déduisent'. Inst. Hautes Etudes Sci. Publ. Math. 2 (1959), 14-60.

[29] — ' 'Ovoîdes à translations', Rendic. Mat. 21 (1962). 37-59.

School of Mathematical Sciences

University of Adelaide

SA 5005

Australia

e-mail: matthew.brown@adelaide.edu.au 
